\title{
ACCIDENTABILIDAD Y REDISEÑO DE LA CARRETERA POROY - URUBAMBA, APLICANDO EL MODELO DE PREDICCIÓN DE ACCIDENTES EN VÍAS RURALES DEL MANUAL NORTEAMERICANO HIGHWAY SAFETY MANUAL 2010
}

\section{Accidentability rate and redesign of the roadway Poroy - Urubamba, based on the accident prediction method used of the Highway Safety Manual 2010.}

\author{
Jean Fernando Pérez Montesinos ${ }^{1, \text { : }}$ \\ ${ }^{1}$ Universidad Andina del Cusco, Cusco, Perú. \\ $\equiv$ jperezm@uandina.edu.pe
}

\begin{abstract}
Resumen
La inexistencia de metodologías en el Perú para la evaluación y rediseño de carreteras e infraestructura vial con alta presencia de accidentes de tránsito no permite salvar vidas humanas mediante la prevención de estos siniestros aplicando la seguridad vial. El objetivo fue calibrar el modelo de predicción de accidentes en condiciones locales (peruanas), siendo el mejor aporte para obtener validez en su aplicación práctica por ingenieros viales locales. La investigación fue desarrollada por profesionales especializados en la ingeniería de transportes y seguridad vial. La metodología tiene el propósito de identificar primero los segmentos de alto riesgo y dar el tratamiento pertinente. Con el modelo de predicción calibrado se procedió a hacer modificaciones en las variables como la geometría para reducir potenciales accidentes. Los resultados fueron satisfactorios, el promedio de calibración obtenido fue de 1.06, que describe errores pequeños pese a limitaciones. Se concluye que, es posible en primer lugar aplicar la metodología norteamericana en el Perú, se redujeron los accidentes hasta un 40\%, siendo este un gran impacto si estimamos la vida humana en el valor dado por la Organización Mundial del a Salud. Esta metodología es el único método de predicción de accidentes a nivel mundial y el más probado en el mundo en los últimos años en varios continentes, permitirá a los ingenieros y políticos peruanos velar por la seguridad vial de una forma científica y demostrada.
\end{abstract}

Palabras clave: seguridad vial, HSM, método predictivo.

\begin{abstract}
There is no Peruvian studies or methodologies to address high presence of accidents in rural and urban highways and roadways in order to safe life and prevent these issues with the use of highway safety. The main purpose was to calibrate a prediction model for accidents for local conditions, through the improvement of geometric design and to show its real applicability. The study was conducted by the student Paul Vargas Meza and its major professor MSc. Jean F. Pérez Montesinos at the Civil Engineering Department who has hand on experience in highway safety methodologies. The method identified first the existence of dangerous segments and provided treatments to solve issues. Once calibrated, it was possible to redesign roadway characteristics to reduce the likelihood of accidents and enhance safety. Research results showed that the estimation of the calibration factor was set to 1.06 for the analyzed segments. Improvements where proposed for the short and midterms. It is possible to conclude that the North American method use can be applied and calibrate for Peruvian local and regional conditions which is proved with a short error in the calibration factor. A huge impact in human life protection was made considering the value stablished by the WHO (World Health Organization). A unique worldwide accident prediction method would allow engineers and politicians improve highway safety with a real a scientific approach. It was the first time that a 50 years old highway safety methodology is tested in Peruvian roadway infrastructures.

Keywords: Highway safety, HSM, predictive method.
\end{abstract}

Citar como: Pérez, J. (2018). Accidentabilidad y rediseño de la carretera poroy - urubamba, aplicando el modelo de predicción de accidentes en vías rurales del manual norteamericano highway safety manual 2010. Rev Yachay, 7(1),339-346.

Recibido: 16-09-2018; Aceptado 07-12-2018

\section{Introducción}

La inseguridad vial es un problema latente a nivel mundial por la tasa alta de accidentes registrados al año. Según el informe sobre la situación mundial de la seguridad vial 2015 afirma que: "El número de accidentes por accidentes de tránsito es de 1.25 millones en el 2013”.

Rev. Yachay volumen (7) Número (1), enero-diciembre 2018 
En el ámbito nacional, según la Policía Nacional del Perú - PNP a través de la Dirección de Estadísticas estima que "cada 7 minutos se produce un accidente de tránsito en la última década (2006 - 2016), ocurren más de 100000 accidentes de tránsito al año y hay alrededor de 80000 heridos por accidentes de tránsito al año". Por otro lado, se observa un crecimiento poblacional acelerado tanto en el sector rural y urbano evidenciando una inadecuada planificación territorial fomentando el desorden, un núcleo urbano enmarañado ignorando el diseño y modos de transporte para una adecuada proyección de espacios públicos. Más aun, el Instituto Nacional de Estadística e Informática INEI indica que, en el año 2017, la población fue de 31 millones 826 mil 18 habitantes, desglosando en 24 millones 735 mil 295 habitantes a nivel urbano y 7 millones 90 mil 723 habitantes a nivel rural.

Asimismo, el parque automotor sufre un incremento continuo de unidades vehiculares motorizadas debido al desarrollo sostenible del país, generando no solo beneficios de manejo económico eficiente sino el desorden vehicular originado por el transporte de servicio urbano y/o rural; para el año 2015 representa 5 millones 245 mil 550 de vehículos registrados, aproximadamente un sexto de la población total del Perú siguiendo una tendencia en los próximos años.

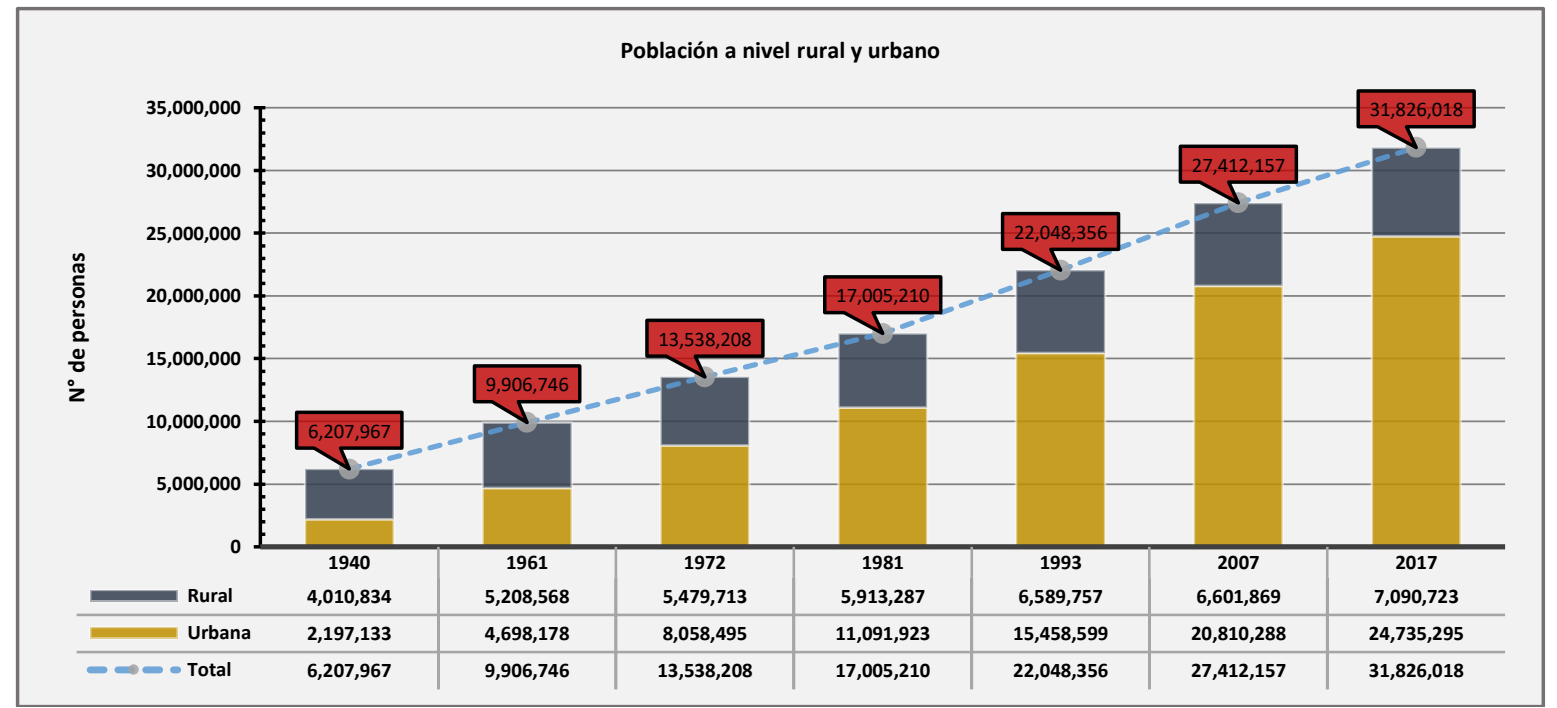

Figura 1. Población a nivel urbano y rural (INEI, 2008-2015. Boletín Especial No 19).

Los accidentes de tránsito son dilemas latentes en el Perú, lo que conlleva a grandes pérdidas socioeconómicas, dado que produce efectos negativos en los habitantes y entorno social, dichos accidentes presentan eventos viales en condiciones fatales, no fatales, pérdidas materiales, entre otros. La mala condición de la infraestructura vial no es ajena a ello, dado que es un factor participativo en la incidencia de accidentes de tránsito siendo escenario de las deficiencias que le atribuyen a esta, poniendo hincapié en el estado en el que se encuentra la vía y/o carencia de señalizaciones tanto horizontales como verticales. La consultora especializada en conservación de infraestructura, Alauda Ingeniería, indica que en el año 2015 los accidentes de tránsito representaron un 3.1\% del PBI dando un costo de S/. 19 '165 millones de nuevos soles.

Por todo ello, la seguridad vial expresa acciones que permiten incrementar la seguridad en las vías o carreteras para disminuir la cantidad de eventos viales que se produce a causa de las múltiples carencias que presenta. Motivo por el cual, las normas peruanas no consideran este aspecto fundamental dado que solo se rigen en la demanda y capacidad vial. Por otra parte, se difiere dos conceptos de seguridad en torno a la ingeniería, la seguridad nominal y la seguridad sustantiva.

La Administración Federal de Carreteras FHWA (siglas en inglés) señala mediante el informe "Estrategias de mitigación para excepciones de diseño" la diferencia entre seguridad nominal y sustantiva indicando que: "La seguridad nominal (línea azul) es una característica de diseño o carretera que cumple con los criterios mínimos o no. Por otro lado, la seguridad sustantiva (línea roja) es el rendimiento de seguridad real a largo plazo o esperado de una carretera; determinado por su experiencia de accidentes medida durante un período de tiempo suficientemente largo, para proporcionar un alto nivel de confianza de que la experiencia de accidentes observada es una representación real de las características de seguridad esperadas de esa ubicación o carretera.” 
Figura 2. Comparación de seguridad nominal y sustantiva (del Informe técnico FHWA, estrategias de mitigación para excepciones de diseño).

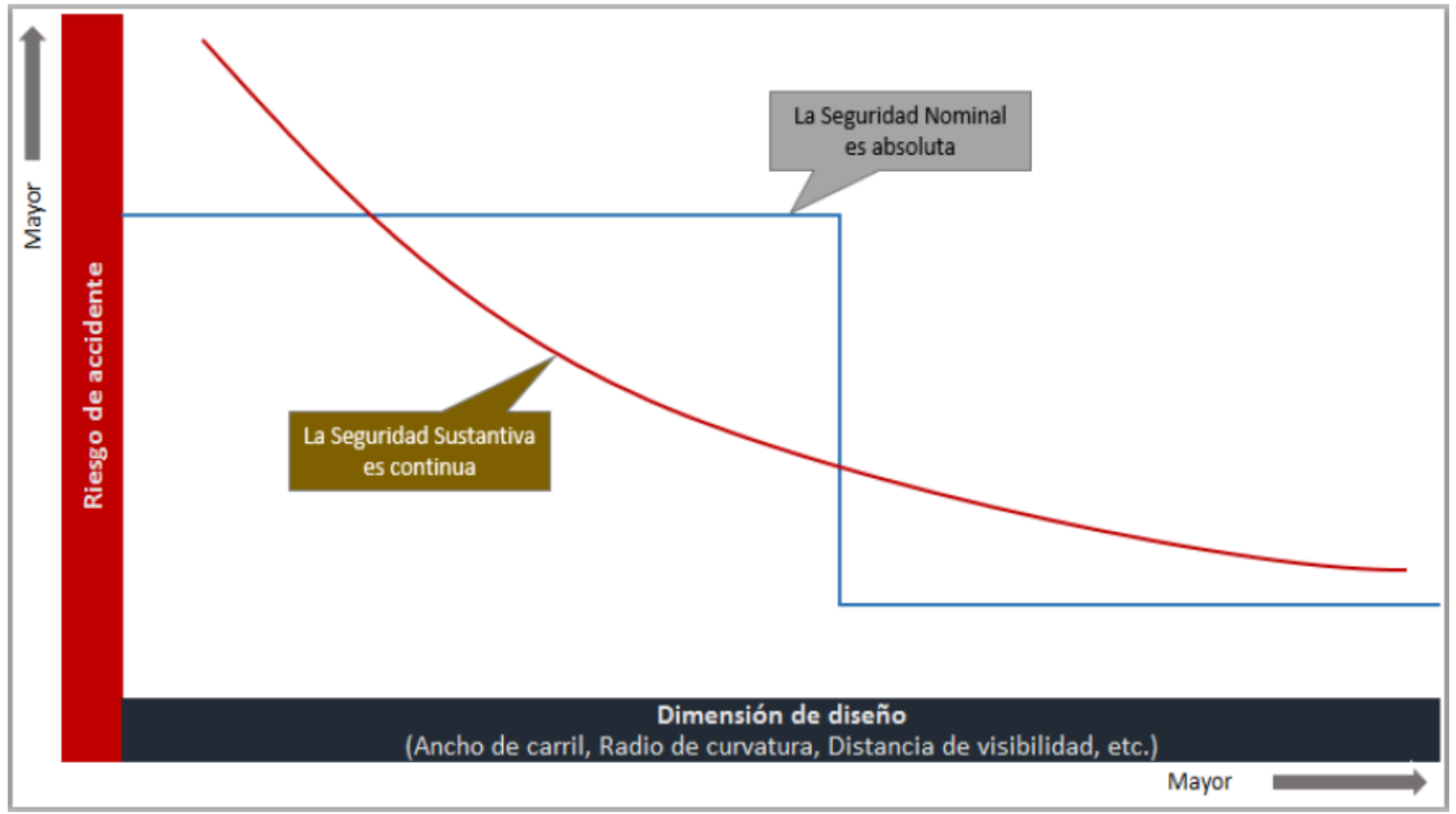

Son muy escasos los estudios relacionados de seguridad vial en el ámbito regional y nacional. En ese entender sobre la base de las consideraciones anteriores, la investigación evaluó la seguridad vial en la carretera nacional PE - 28 F mediante un análisis que determine los aspectos técnicos, elementos defectuosos que generan la alta incidencia de accidentes de tránsito pudiendo formular la siguiente pregunta: ¿Cómo inciden los componentes geométricos, la intensidad de tránsito y sistemas de control de tránsito en la accidentabilidad ocasionada en la carretera nacional PE - 28 F? generando la hipótesis que indica: Los componentes geométricos, intensidad de tránsito y sistemas de control de tránsito inciden directamente en la accidentabilidad en la carretera nacional PE - 28 F. Para tal aseveración se tendrá que analizar las características viales de la carretera entorno a la seguridad que presenta en condiciones actuales, limitadas a la información obtenida por autoridades competentes caso PNP, MTC - Provías Nacional y el manual de seguridad vial HSM.

\section{Materiales y métodos}

El presente artículo proviene de la tesis de investigación para optar al Título Profesional de Ingeniero Civil, en la Escuela Profesional de Ingeniería Civil de la Universidad Andina del Cusco.

La investigación presenta el enfoque cuantitativo de nivel descriptivo y el método deductivo - cuantitativo, ya que se plantea inicialmente la hipótesis general, como específicas de modo que en base a los resultados obtenidos se afirma o se refuta la suposición indicada. El diseño fue no experimental, debido a que se llega a analizar las situaciones ya existentes, teniendo en cuenta de que las variables independientes no llegaron a ser manipuladas, siendo observados en su forma natural. Asimismo, presenta un diseño longitudinal puesto que se llega hacer la recolección de datos en diferentes periodos.

El trabajo de investigación desarrolló dos poblaciones: primera población, establecido por toda la ruta de la carretera nacional PE - $28 \mathrm{~F}$ de $44.990 \mathrm{~km}$ y la segunda población, constituida por los informes policiales referido a los accidentes de tránsito de cada jurisdicción comprendida en la carretera nacional PE - 28 F pertenecientes a los últimos 5 años (2013-2017). De igual forma, la muestra contempla los dos elementos representativos de la población.

El estudio empleó el método de muestreo probabilístico, debido a que las muestras tienen la misma opción de ser escogidos al azar o en forma aleatoria en los miembros de la población. Asimismo, se considera que el muestreo es integral dado que se considera toda la longitud total de la vía. 
Los instrumentos de recolección de datos fueron formatos específicos empleados en campo y posteriormente filtrados en gabinete, de los cuales se tiene: Aforo vehicular, datos geométricos de la infraestructura vial, datos de los sistemas de control de tránsito e informes de accidentes de tránsito. Los instrumentos de ingeniería utilizados para la toma de datos fueron: GPS - submétrico, Wincha topográfica, eclímetro, software de ingeniería e incluyendo cámara - filmadora, DVR - digital video recorder y una unidad vehicular.

El trabajo de investigación fue desarrollado tomando las variables independientes relacionadas a la accidentabilidad que presenta la carretera de estudio, los componentes geométricos conformado por ocho indicadores, la intensidad de tránsito compuesta por el volumen vehicular del sitio y los sistemas de control de tránsito conformado por cinco indicadores atribuyendo la generación de accidentes viales a causa de estas.

La medida de precisión de la metodología del Manual de Seguridad Vial norteamericano HSM 2010 se cuantifica mediante el denominado factor de calibración, que es el ajuste o cercanía a los valores reales de campo, es decir accidentes que se han registrado en las comisarías versus número de accidentes predichos por la metodología. En el caso del estudio este valor fue de 1.06, lo que prueba que se tiene un error del $6 \%$ y completamente aplicable a la realidad peruana.

\section{Resultados}

En relación a los componentes geométricos frente a la accidentabilidad, se mostró una composición de tramos tangente de 29.63 km representando el $65.86 \%$ y $15.36 \mathrm{~km}$ en curvas, señalando $34.14 \%$ de toda la vía de estudio. Respecto a los segmentos tangentes la mayor incidencia de eventos viales señala una cifra superior a 1.50. En referencia a los segmentos curva, todos presentan una cifra superior a 1.00 indicando que los factores son elevados debido al incumplimiento de parámetros mínimos de diseño predominando los segmentos curva a causa de la falta de radios mínimos y la longitud de curvatura reducida en determinados segmentos; destacando en el sector de Maras la mayor accidentabilidad; dado que, se observa la constante incidencia de eventos viales específicamente en la cuesta de la entrada al poblado de Urubamba preservando un diseño sinuoso.

Figura 3. Componentes geométricos frente a la accidentabilidad

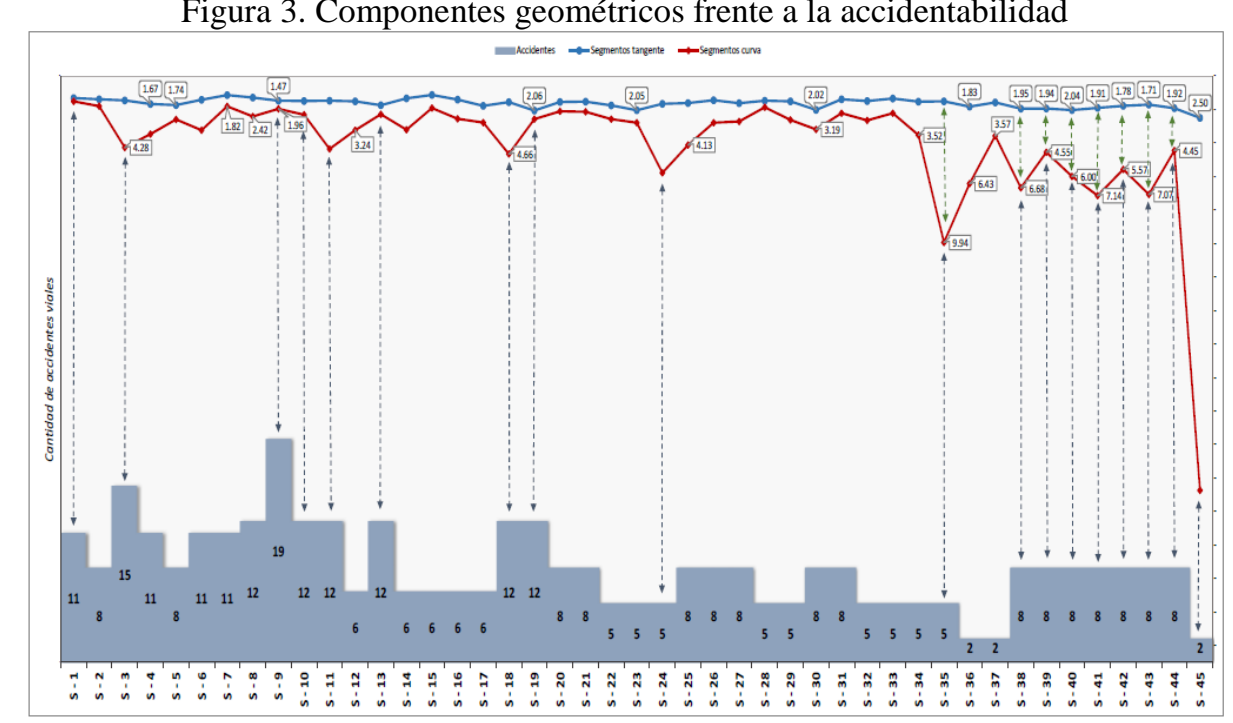

En relación a la intensidad de tránsito frente a la accidentabilidad, presenta variaciones a lo largo del día con picos altos en horas de la mañana entre las 07:00 - 10:00 horas y en la tarde entre las 16:00 - 19:00 horas. La cuantificación del tránsito promedio diario fue de $6446 \mathrm{veh} /$ día apreciando un crecimiento superior del 90\% respecto al año 2013, sufriendo una atracción de vehículos por actividades de la zona (turismo, factores laborales, factores económicos, demográficos) y población vinculada a la carretera que ocupa labores rudimentarias del sector.

En relación a los sistemas de control de tránsito frente a la accidentabilidad, se señaló mediante el modelo, la implementación de los factores de modificación de accidentes manifestando la situación actual vinculada a los sistemas de control de tránsito (valga la redundancia) frente a la incidencia de accidentes viales, dichos factores se establecen en segmentos tangente como en curvas, observando el decrecimiento a causa de la aplicación en condiciones óptimas para cada segmento, generando mayor seguridad vial en la carretera.

Rev. Yachay volumen (7) Número (1), enero-diciembre 2018 
Figura 4. Sistemas de control de tránsito frente a la accidentabilidad

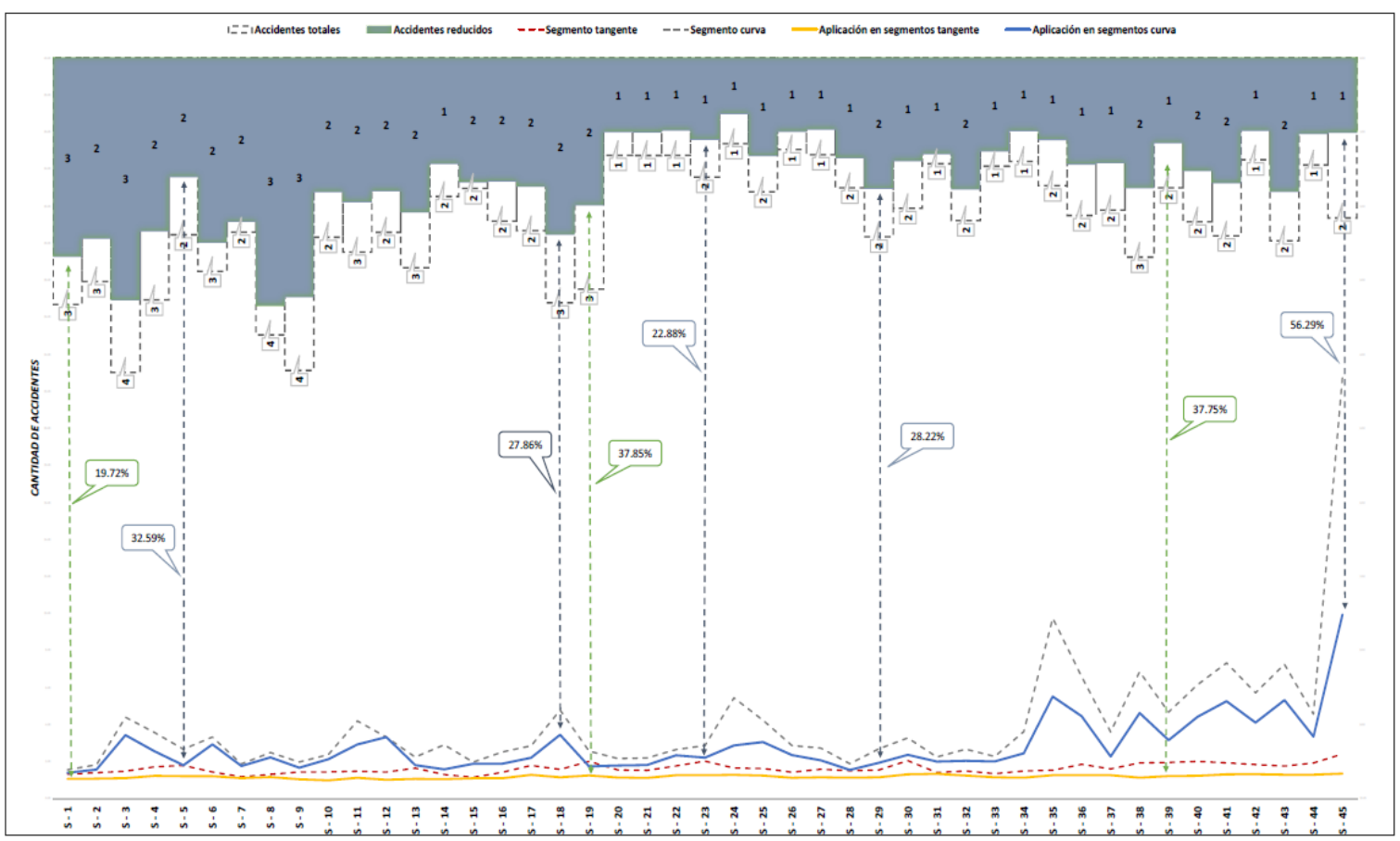

En relación a la propuesta de solución frente a la accidentabilidad, se dio con la finalidad de aminorar los accidentes viales a corto y mediano plazo en la carretera nacional PE - 28 F. Considerando esta hipótesis se hizo uso de la metodología del HSM para vial rurales de dos carriles modificando siete indicadores instaurados; y así, predisponer la cantidad de accidentes esperados según sea la jurisdicción correspondiente durante los próximos 15 años. En la figura 5 se aprecia la reducción de eventos viales según sea el sector correspondiente.

De los resultados obtenidos en referencia al factor de calibración, se observó factores de modificación de accidentes elevados lo que conlleva al incremento de accidentes viales tanto en segmentos tangente como en curva. Dando la interpretación de que los valores de 1.06 y 1.38 subestiman la cantidad de accidentes en un $6 \%$ y $38 \%$ respectivamente. Por otro lado, el valor de 0.73 sobrestima la cantidad de accidentes en un 73\%; dado que, presentan indicadores altos siendo de mayor incidencia en segmentos curva. Sin embargo, se puede inferir que las cifras resultantes se encuentran estrechamente vinculadas con cantidad de segmentos a analizar, teniendo como limitante la información de accidentes viales en forma detallada para una mejor precisión.

Al detectar los elementos geométricos que generan accidentes y dificultan la circulación vehicular como geometrías sub-diseñadas conforme a la norma de diseño geométrico de carreteras, se procedió a cambiar los valores de dicha geometría y estas se presentan en la alternativa de solución. En la figura 5, se observa el impacto en cuanto a reducción de accidentes generados por la modificación geométrica en elementos como radios de curvatura, pendientes longitudinales, ancho de carril, señalización y señalización. De todos los elementos geométricos modificados el tamaño del radio de curvatura es el que más impacto tiene en la reducción de accidentes, seguido por el control de pendientes y ancho de la sección de la vía expresado en ancho de carril y bermas.

Figura 5. Reducción de eventos viales según jurisdicción- Alternativa 


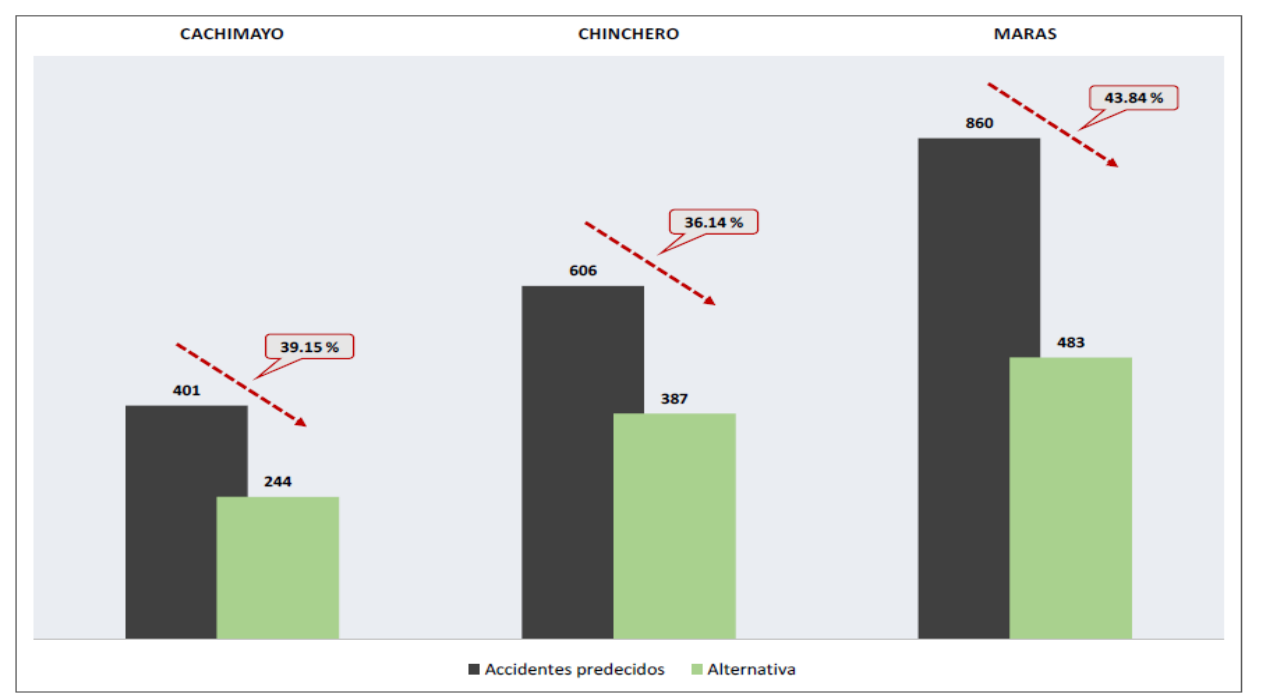

\section{Discusión}

\section{01. ¿Se aplicaron los criterios de seguridad nominal y seguridad sustantiva en la presente investigación?}

Respuesta: Sí, durante la evaluación de la carretera nacional PE - 28 F se tomó en consideración todos los conceptos indicados por las normas peruanas y referentes al diseño de carreteras sean: DG - 2018, Manual de carreteras de INVIAS, Manual de dispositivos de control de tránsito y otras fuentes relacionadas a esta, siendo vinculados a la seguridad nominal; Por otra parte, se empleó la metodología del Highway Safety Manual HSM 2010 que involucra la situación real de las características de la infraestructura vial con la accidentabilidad relacionando cada uno de los componentes generando un modelo que describe el comportamiento en cada una de ellas describiendo una seguridad sustantiva.

\section{D.02. ¿De qué depende la precisión del modelo Highway Safety Manual HSM 2010 empleado en la presente investigación?}

Respuesta: La precisión depende del acceso y la disponibilidad de datos que involucra la metodología HSM, un adecuado análisis depende de la información exacta de aquellos sectores donde presenta mayor cantidad de eventos viales. La información otorgada por los diferentes órganos competentes no es muy detallada, e incluso los datos no son confiables, lo que conlleva a datos no precisos, sujeto a errores. Para la presente investigación se facilitó, por medio Policía nacional del Perú PNP, la cantidad de los accidentes de tránsito en condiciones fatales y no fatales anuales, siendo necesario la información detallada y exacta de aquellos sectores con mayor demanda de accidentes para la oportuna intervención.

D.03. ¿A qué se debe la diferencia del factor de calibración obtenido en la presente tesis con los factores hallados en los antecedentes mencionados anteriormente?

Respuesta: El factor de calibración que se obtuvo en la presente investigación fue de 1.06 jurisdicción de Cachimayo, 1.38 jurisdicción de Chinchero y 0.73 jurisdicción de Maras, interpretando que cada sector presenta diferentes comportamientos según las zonas instauradas paralelas a la vía. De igual forma, el factor de calibración a nivel local en la carretera PE - 3S tramo Cusco - Urcos, indicado por la autora, Fabiola Amelia Canaza Cabrera, fue de 1.32 señalando la predisposición a eventos viales en un $32 \%$ para años posteriores. Asimismo, en la investigación internacional de María Graciela Berardo, se señala la calibración de 0.85 para un tramo de 15 km, deduciendo una reducción del 15\% de eventos viales a futuro; Por lo tanto, el factor de calibración es solo para las condiciones del sitio de estudio que presenta características específicas.

\section{D.04. ¿Un buen diseño geométrico garantiza la seguridad vial de una carretera?}

Respuesta: No, si bien es cierto las normas y/o reglamentos tienen como objetivo garantizar los parámetros o criterio mínimos que involucra una infraestructura vial en la ejecución o mejoramiento, por ello no determina umbrales sólidos de seguridad. Además, no se interpreta la aplicación de normas como el respaldo de la seguridad vial. Por ende, es importante realizar estimaciones de predicciones de accidentes de tránsito en un proyecto de inversión para determinar si la vía en sí, es segura; si fuera el caso de no estar con los 
lineamientos correctos se procede a la intervención a través de modificaciones para dar un adecuado diseño; Por lo tanto, es necesario realizar una evaluación de la seguridad nominal conjuntamente con la seguridad sustantiva señalando un análisis integral con la finalidad de preservar el bienestar de los usuarios de la vía.

\section{Conclusiones}

I. De las figuras 3 y 4 se evidencia los segmentos de mayor deficiencia, tanto en los componentes geométricos como en los sistemas de control de tránsito que presentan un elevado factor en tangente como en curvas. Respecto a la intensidad de tránsito, este influye en los accidentes viales dado que el incremento de unidades vehiculares incita a producir eventos viales. Así mismo, es una variable vinculante en el modelo empleado; sin embargo, no se asevera una relación directa señalando que sí existe mayor volumen vehicular, lo que genera mayor cantidad de accidentes viales. Poniendo hincapié de los demás factores externos que no son incluidos en la metodología HSM como: exceso de velocidad, deterioro del estado del pavimento o carpeta de rodadura (ahuellamiento, piel de cocodrilo, entre otros), carencia de sobre anchos en curvas, factores climatológicos, señalización horizontal y señalización vertical indicando la probabilidad de que ocurra un evento vial a causa de estas.

II. En el análisis se determinaron los componentes geométricos, tanto segmentos tangentes como curvas, que presenta la carretera a través del modelo del HSM por medio de los factores de modificación de accidentes indicando factores elevados en referencia los segmentos en curva lo que atribuye a la generación de accidentes, concluyendo que la vía de estudio presenta valores de radio mínimo por debajo de lo establecido por normas y/o reglamentos generando la accidentabilidad en la carretera nacional.

III. Se estableció que el incremento de la intensidad de tránsito influye en la generación de accidentes. Así mismo, es una variable instaurada en el modelo empleado HSM 2010. No obstante, la incidencia de accidentes viales se puede atribuir a diferentes factores externos.

IV. Se verificó la aplicación de los sistemas de control de tránsito en los segmentos establecidos incidiendo en los accidentes predispuestos para el año 2018, resaltando el empleo de los factores de modificación de accidentes en segmentos tangente y curvas, concluyendo que la presente carretera de estudio muestra una incidencia del $25.19 \%$ de eventos viales respecto a la carencia de dichos sistemas.

V. Se estimó la influencia de la propuesta de solución efectuando una intervención a corto y mediano plazo, indicando la reducción de eventos viales en consecuencia de los cambios relativos en los componentes geométricos y sistemas de control de tránsito, mostrando un decrecimiento del $40.33 \%$ durante los próximos 15 años. Destacando el incremento de la seguridad vial en los sectores de Cachimayo, Chinchero y Maras de la región del Cusco.

VI. El factor de calibración describe el comportamiento de una infraestructura vial en referencia a los parámetros de diseño de carreteras, conformado por fuentes norteamericanas basados en manuales y estudios realizados en vías que relacionan la atribución de eventos viales.

\section{Agradecimiento}

A la Universidad Andina del Cusco, por la oportunidad de desarrollar esta investigación y por promover el desarrollo de nuevos aportes a la Ingeniería de Transportes y la Seguridad Vial.

\section{Referencias}

American Association of State Transportation Officials. (2010). Highway Safety Manual, AASHTO. Washington D.C.

Canaza Cabrera, F. A. (2016). Propuesta de alternativas de mejoramiento de la carretera Cusco-Puno tramo Cusco-Urcos aplicando la metodologia de inspeccion de seguridad vial y prediccion de accidentes viales segun el HSM. Cusco: Universidad Andina del Cusco.

Rev. Yachay volumen (7) Número (1), enero-diciembre 2018 
Graciela Berardo, M. (2015). Aplicación del modelo de predicción de accidentes viales del HSM (2010) en camino rural de dos carriles en Brasil. Revista Facultad de Ciencias Exactas, Fisicas y Naturales, 51.

Instituto Nacional de Estadística e Informática INEI. (2008). Perfil Sociodemografico del Peru. Lima.

Ministerio de Transportes y Comunicaciones MTC. (2016). Manual de Dispositivos de Control del Tránsito Automotor para Calles y Carreteras. Lima, Peru.

Ministerio de Transportes y Comunicaciones MTC. (2018). Manual de Carreteras, Diseño Geometrico DG 2018. Lima, Peru.

Organización Mundial de la Salud. (2015). Informe sobre la situacion mundial de la seguridad vial 2015. Suiza: Inis Communication.

U.S. Department of Transportation. (s.f). Access Management (Driveways). FHWA-SA-14-080, Federal Highway Administration. Obtenido de https://goo.gl/7t62n2 